\title{
Inclusion and Analysis of Older Adults in RCTs
}

\author{
Donna M. Zulman, MD, MS, Jeremy B. Sussman, MD, MS, and Rodney A. Hayward, MD
}

Robert Wood Johnson Foundation Clinical Scholars Program, University of Michigan, Ann Arbor, MI, USA.

$\mathrm{J}$ Gen Intern Med 26(8):832

DOI: $10.1007 / \mathrm{s} 11606-011-1706-9$

(c) Society of General Internal Medicine 2011

Authors' Reply: We appreciate the comments of Van de Water and colleagues. Their examination of the impact of cancer trial eligibility criteria is an example of how common age-related exclusion criteria-criteria that we found to be prevalent in our systematic review of clinical

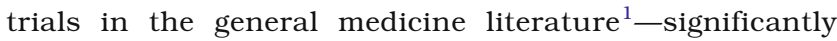
limit the generalizability of study results to many typical older patients. We also agree with their observation that expanding trial eligibility criteria is not without challenges, and that in some circumstances there will be unavoidable barriers to designing a randomized controlled trial (RCT) that adequately informs certain aspects of care for older adults. In these instances, as Van de Water and colleagues note, many insights can be gained from well-designed cohort and case-control studies. ${ }^{2}$

Our study revealed, however, that even when RCTs do examine the effectiveness and safety of treatment in older adults, they frequently do not use appropriate statistical methods to examine heterogeneity (variability) in treatment effects by age. ${ }^{3,4}$ In particular, few trials explore whether older adults respond differently to a treatment due to their age, or due to their overall risk for disease-related outcomes and

Published online April 5, 2011 complications (a risk that is often influenced by comorbidities, health status, and other characteristics). This information can be of great value to clinicians who are trying to determine whether an individual patient will benefit from a given therapy. Our knowledge base about treatment effects in older adults will, therefore, benefit not only from rigorous observational research and from RCTs that minimize age-related exclusion criteria, but also from more widespread use of statistical approaches that allow us to investigate whether age in itself, or other characteristics prevalent among older adults, explain heterogeneity in treatment effects among our older patients.

Corresponding Author: Donna M. Zulman, MD, MS; Robert Wood Johnson Foundation Clinical Scholars Program, University of Michigan, 6312 Medical Sciene Building 1, 1150 W. Medical Center Drive, Ann Arbor, MI 48109, USA (e-mail: dzulman@umich.edu).

\section{REFERENCES}

1. Zulman DM, Sussman JB, Chen X, Cigolle CT, Blaum CS, Hayward RA. Examining the evidence: a systematic review of the inclusion and analysis of older adults in randomized controlled trials. J Gen Intern Med. 2011. doi: 10.1007/s11606-010-1629-x.

2. Nallamothu BK, Hayward RA, Bates ER. Beyond the randomized clinical trial: the role of effectiveness studies in evaluating cardiovascular therapies. Circulation. 2008;118(12):1294-303.

3. Kent DM, Rothwell PM, Ioannidis JP, Altman DG, Hayward RA. Assessing and reporting heterogeneity in treatment effects in clinical trials: a proposal. Trials. 2010;11:85.

4. Kent DM, Hayward RA. Limitations of applying summary results of clinical trials to individual patients: the need for risk stratification. JAMA. 2007;298(10):1029-12. 